\title{
Atomic-scale characterization of nitrogen-doped graphite: Effects of dopant nitrogen on the local electronic structure of the surrounding carbon atoms
}

\author{
Takahiro Kondo, ${ }^{1}$ Simone Casolo, ${ }^{2}$ Tetsuya Suzuki, ${ }^{1}$ Taishi Shikano, ${ }^{1}$ Masataka Sakurai, ${ }^{1}$ Yoshihisa Harada,, 4 \\ Makoto Saito, ${ }^{3, *}$ Masaharu Oshima, ${ }^{3,4}$ Mario Italo Trioni, ${ }^{5}$ Gian Franco Tantardini, $, 2,5$ and Junji Nakamura ${ }^{1, \dagger}$ \\ ${ }^{1}$ Faculty of Pure and Applied Sciences, University of Tsukuba, 1-1-1 Tennodai, Tsukuba, Ibaraki 305-8573, Japan \\ ${ }^{2}$ Dipartimento di Chimica Fisica ed Elettrochimica, Università degli Studi di Milano, via Golgi 19, 20133 Milan, Italy \\ ${ }^{3}$ Department of Applied Chemistry, The University of Tokyo, 7-3-1 Bunkyo-ku, Tokyo 113-8656, Japan \\ ${ }^{4}$ The University of Tokyo Synchrotron Radiation Research Organization, 7-3-1 Bunkyo-ku, Tokyo 113-8656, Japan \\ ${ }^{5}$ CNR National Research Council of Italy, ISTM, via Golgi 19, 20133 Milan, Italy
}

(Received 4 January 2012; published 23 July 2012)

\begin{abstract}
We report on the local atomic and electronic structures of a nitrogen-doped graphite surface by scanning tunneling microscopy, scanning tunneling spectroscopy, x-ray photoelectron spectroscopy, and first-principles calculations. The nitrogen-doped graphite was prepared by nitrogen ion bombardment followed by thermal annealing. Two types of nitrogen species were identified at the atomic level: pyridinic- $\mathrm{N}$ ( $\mathrm{N}$ bonded to two $\mathrm{C}$ nearest neighbors) and graphitic- $\mathrm{N}$ ( $\mathrm{N}$ bonded to three $\mathrm{C}$ nearest neighbors). Distinct electronic states of localized $\pi$ states were found to appear in the occupied and unoccupied regions near the Fermi level at the carbon atoms around pyridinic- $\mathrm{N}$ and graphitic- $\mathrm{N}$ species, respectively. The origin of these states is discussed based on experimental results and theoretical simulations.
\end{abstract}

DOI: 10.1103/PhysRevB.86.035436

PACS number(s): 81.05.ue, 07.79.Fc, 73.22.Pr, 81.05.uf

\section{INTRODUCTION}

Nitrogen doping in graphitic materials such as graphene and carbon nanotubes has been reported to modify physical and chemical properties. ${ }^{1-8}$ Various applications of $\mathrm{N}$ doped graphite materials have also been reported, such as in biosensors, ${ }^{9}$ fuel cells, ${ }^{10-15}$ capacitors, ${ }^{16}$ electronic devices, ${ }^{17-19}$ and spin filter devices. ${ }^{20,21}$ By X-ray photoelectron spectroscopy (XPS), the nitrogen species in N-doped graphite materials ${ }^{12,13,15,19,22-26}$ have been identified mainly as pyridinic-N (characterized by a binding energy of $\sim 398.5 \mathrm{eV}$ ), pyrrolic-N (400.5 eV), and graphitic-N or quaternary-N (401.2 eV). ${ }^{6,27-29}$ However, a detailed picture of the local electronic modifications in $\mathrm{N}$-doped graphite induced by each of $\mathrm{N}$ atoms has not been clarified.

The electronic structure near the Fermi level $\left(E_{F}\right)$ of the graphite surface is known to be modified by the defect such as a point vacancy, where localized $\pi$ electronic states are reported to form at the neighboring $\mathrm{C}$ atoms and propagate anisotropically around the defect even to a few nanometers away from the defect due to the perturbation of the $\pi$ conjugated system of graphite. ${ }^{30}$ It is thus expected that the nitrogen dopants in N-doped graphite modify the electronic structure near $E_{F}$ and that the modified electronic structure is different from that of the point vacancy defect because the nitrogen bonds to some carbon atoms. It is also expected that the modified electronic structure is different depending on the types of $\mathrm{C}-\mathrm{N}$ bonding configurations.

Here, we report on the atomic-scale characterization of a $\mathrm{N}$-doped graphite surface by scanning tunneling microscopy (STM), scanning tunneling spectroscopy (STS), XPS, and first-principles calculations based on the density functional theory (DFT). Two types of nitrogen species, graphitic-N and pyridinic-N, were identified at the atomic scale, and the effect of each nitrogen species on the local electronic structure of the surrounding carbon atoms is discussed based on experimental results and theoretical simulations.

\section{EXPERIMENTAL DETAILS}

$\mathrm{N}$-doped graphite was prepared in situ in all experiments reported here as follows: A fresh highly oriented pyrolytic graphite (ZYA grade HOPG, Panasonic Inc.) sample was first cleaved in air using an adhesive tape and was placed in an ultra-high-vacuum (UHV) chamber. Subsequently, it was annealed at $900 \pm 50 \mathrm{~K}$. The sample was then bombarded by nitrogen ions of $200 \mathrm{eV}$ (or $500 \mathrm{eV}$ ) at $300 \mathrm{~K}$ and normal incidence using a commercial ion gun (5-kV ion gun, Anelva). The total ion doses on the sample were set to be lower than 0.2 $\times 10^{13}$ ions $/ \mathrm{cm}^{2}$, which were measured independently using a Faraday cup (for the XPS experiment, we didn't measure the ion current). After the bombardment, the sample was annealed at $900 \pm 50 \mathrm{~K}$ for $300 \mathrm{~s}$. Based on the STM observations, the irradiated ions are found to form defects in the sample with high efficiency (see, Fig. S1 in the Supplemental Material ${ }^{47}$ ). Some nitrogen atoms were implanted into the bulk of the graphite, and as a result, the STM image showed peculiar characteristics, even after further cleavage in air followed by annealing in UHV. The sample temperature during heating was measured using an infrared thermometer.

XPS spectra were measured at $160 \mathrm{~K}$ in the UHV chamber with a base pressure of $3 \times 10^{-10}$ torr at BL27SU in SPring- 8 in Hyogo, Japan. The excitation photon energy used in this work was $700 \mathrm{eV}$. The $\mathrm{x}$-ray excitation and photoelectron detection angles were set to $45^{\circ}$ with respect to the sample normal. The total energy resolution was $\sim 0.24 \mathrm{eV}$. Energy calibration was performed using the $\mathrm{Au} 4 f_{7 / 2}$ photoemission at $84.0 \mathrm{eV}$.

STM and STS measurements were performed in the UHV chamber (USM-1200, Unisoku) with a base pressure of $1 \times$ $10^{-10}$ torr. A commercial STM controller (SPM-1000, RHK Technologies) was used. The sample was cooled to $5.3 \mathrm{~K}$. The surface morphology and the surface electronic structure were then examined by STM and STS, respectively, with a PtIr tip (Pt:Ir = 8:2). STM images were recorded in the constant-current mode. STS measurements were carried out 
by measuring the differential conductance $(d I / d V)$ by a lock-in detection technique using $1.0-\mathrm{kHz}$ alternating-current modulation of the sample bias voltage with an amplitude of $20 \mathrm{mV}$. $d I / d V$ reflects features in the local density of states (LDOS) of the surface at the position of the STM tip, where $d I / d V$ at negative and positive sample biases correspond to LDOS of occupied and unoccupied states, respectively, and $0 \mathrm{~V}$ corresponds to the Fermi level. ${ }^{31}$ We used a small set current of 50-200 pA to minimize the effect of the tip on the tunneling spectrum. The STS spectra shown here represent the average of 10 spectra measured at the same position.

\section{THEORETICAL DETAILS}

The electronic structure and density of states (DOS) of the $\mathrm{N}$-doped graphite sample were studied using DFT.

In theoretical simulations, an orthorhombic cell was preferred to a hexagonal one to avoid any (fictitious) band gap due to superlattice effects. ${ }^{32}$ The supercell was made by graphene layers of $96 \mathrm{C}$ atoms each, and defects were introduced in a single layer or in a three-layer cell, on top of which a $20-\AA$ vacuum was used to avoid interaction between periodic replicas. The graphene layers were kept fixed at the experimental interlayer distance because of the well-known failure of DFT in dealing with dispersion forces. After careful testing, we found that three graphene layers (for a total of 288 atoms) are sufficient to obtain converged properties.

Equilibrium geometries for $\mathrm{N}$-doped graphite were first obtained using a plane wave DFT, as implemented in the Vienna ab initio simulation package code ${ }^{33}$ by using a $500 \mathrm{eV}$ energy cutoff and a $3 \times 3 \times 1 \Gamma$-centered k-point mesh. The relaxation of the atomic position was stopped when the highest force acting on each ion was lower than $0.03 \mathrm{eV} / \AA$.

Core electrons, considered frozen here, were included through projector augmented wave potentials, ${ }^{34,35}$ while small (0.05 eV) Gaussian smearing was used to improve electronic convergence. Exchange and correlation was taken into account according to the gradient-corrected Perdew-BurkeErnzerhof ${ }^{36}$ functional. Later, atomic positions were optimized again with the Spanish initiative for electronic simulations with thousands of atoms (SIESTA) $\operatorname{code}^{37}$ using a double- $\zeta$ plus polarization basis set, obtaining only negligible differences.

STM images and STS spectra were all simulated using SIESTA. Accurate LDOS were computed from the electronic ground state on a $100 \times 100 \times 1 \mathrm{k}$-point mesh, and as STS simulations, we show the DOS projected on $p_{z}$ orbitals of the topmost layer only, which are the ones mainly probed by the STM tip. The Fermi energy was set to the Dirac point, because this is the limit for diluted defects. Simulated STM images were obtained within the Tersoff-Hamann approach ${ }^{31}$ (constant-current mode) for a doped single-layer graphene system that was found sufficient to give good agreement with the experiment. In this work, the spatial distribution of the selected electron densities (obtained from the wave function square modulus) are shown as isosurface plots from a top view.

\section{RESULTS AND DISCUSSION}

The XPS and STM measurements of N-doped graphite are described first in Sec. IV A. The local electronic structures (a)

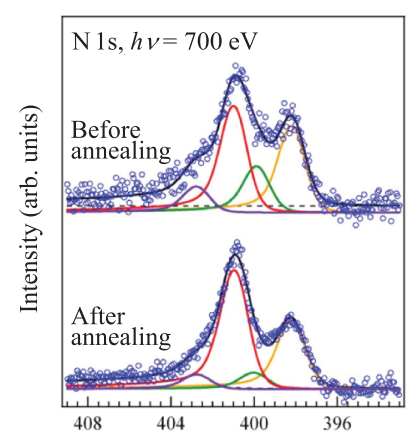

(c)

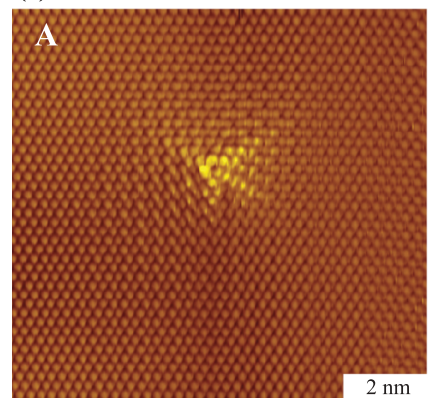

(b)

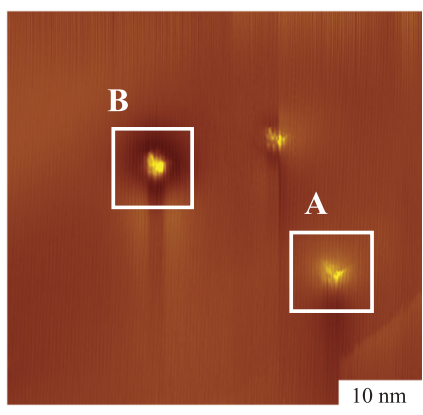

(d)

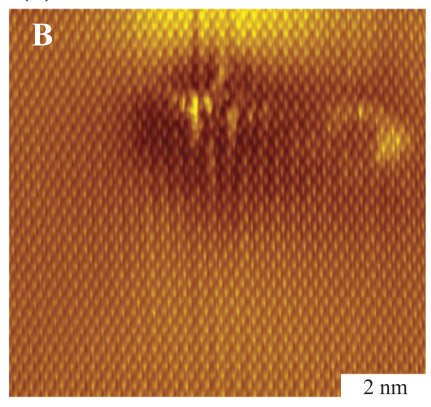

FIG. 1. (Color online) (a) N 1s XPS spectra of the graphite surface after nitrogen ion bombardment at $200 \mathrm{eV}$. The results before and after annealing at $900 \pm 50 \mathrm{~K}$ for $300 \mathrm{~s}$ are shown. Deconvoluted components are pyridinic-N (398.5 eV; before: 32.6\%, after: $31.4 \%)$, pyrrolic-N (399.9 eV; before: 17.4\%, after: 7.6\%), graphitic-N (401.1 eV; before: $40.1 \%$, after: $54.3 \%$ ), and oxide-N (403.2 eV; before: $9.8 \%$, after: $6.8 \%$ ). (b) Typical STM topographic image of the N-doped graphite surface $\sim 5.3 \mathrm{~K}$ (scan size: $49.45 \times 46.97 \mathrm{~nm}^{2}$, tunneling current $I_{t}=179 \mathrm{pA}$, sample bias $V_{s}=98.8 \mathrm{mV}$ ). (c) and (d) STM topographic images of regions A and B in Fig. 1(b), respectively (in both cases, scan size: $9.88 \times 9.26 \mathrm{~nm}^{2}, I_{t}=97.5 \mathrm{pA}, V_{s}=$ $-109 \mathrm{mV}$ ).

of the surface near the Fermi level around the characterized dopant nitrogen species, pyridinic-N and graphitic-N, are then discussed in Secs. IV B and IV C, respectively, based on the experimental results and DFT calculations.

\section{A. XPS and STM measurements}

Figure 1(a) shows N 1s XPS spectra of the N-doped graphite sample, where the results are shown for the surface bombarded with nitrogen ions at $200 \mathrm{eV}$ before and after annealing at $900 \mathrm{~K}$. The total amount of nitrogen with respect to that of carbon decreased from 3.1 to 2.7 at\% after annealing, where the amounts were estimated by simply calculating the ratio between $\mathrm{C} 1 \mathrm{~s}$ and $\mathrm{N}$ 1s peak areas and by considering the sensitivity factors; i.e., nitrogen atoms were assumed to be doped uniformly into deeper graphite layers. The decrease in the amount of nitrogen during annealing up to $900 \mathrm{~K}$ was possibly due to the desorption of $\mathrm{N}_{2}$ or $\mathrm{CN}$. Four $\mathrm{N} 1 \mathrm{~s}$ peak components at 398.5, 399.9, 401.1, and $403.2 \mathrm{eV}$ have been assigned to pyridinic-N (N connected to two $\mathrm{C}$ ), pyrrolic-N (N part of a pentagon ring connected to two $\mathrm{C}$ ), graphitic-N (N connected to three $\mathrm{C}$ ), and oxide-N species, respectively. ${ }^{6,27-29}$ Here, the energy position for pyrrolic- $\mathrm{N}$ is known to be similar to that for cyanide- $\mathrm{N}$ ( $\mathrm{N}$ connected to a single $\mathrm{C}, 399.5 \mathrm{eV}$ ) and 
amine- $\mathrm{N}$ ( $\mathrm{N}$ connected to a single $\mathrm{C}$ and two $\mathrm{H}, 399.4 \mathrm{eV}$ ), ${ }^{27}$ possibly also contributing to the same peak. In our samples, pyridinic- $\mathrm{N}$ and graphitic- $\mathrm{N}$ were the dominant components, as shown in Fig. 1(a). The same two peak components have been reported as dominant for nitrogen-doped graphite or graphene prepared by the ion bombardment method, also for different collision energies $(0.1-6.0 \mathrm{keV})$, different annealing temperatures, or both. ${ }^{23,25,26,38}$ However, the ratio of the peak intensities depends on the ion collision energy, flux of ions, and annealing temperature. In Fig. 1(a), graphitic-N is the largest component $(40.1 \%)$ after nitrogen ion bombardment at $200 \mathrm{eV}$. After annealing to $900 \mathrm{~K}$, the relative intensity of this peak increased (54.3\%), suggesting that the other species were converted to graphitic-N. It has been reported that the relative amount of pyridinic- $\mathrm{N}$ is comparable to that of graphitic-N when the sample is prepared with higher energies (a few kiloelectron volts) and more intense ion fluxes of nitrogen. ${ }^{23,25,26,38}$ This suggests that the graphitic-N-rich surfaces can be obtained with small doses and low energies of nitrogen ion.

A typical STM image of the $\mathrm{N}$-doped graphite surface is shown in Fig. 1(b). Two types of bright species were observed with an average diameter of $\sim 4 \mathrm{~nm}$ : one was surrounded by a bright region (type A), and the other was surrounded by a dark region (type B). Because it is generally difficult to identify surface atom elements by STM alone, we have carried out the characterization by combining STS analysis and DFT calculations. We show here that type-A and type-B defects can be assigned to pyridinic- $\mathrm{N}$ species with a single-atom vacancy and graphitic-N species, respectively. These indeed correspond to the two dominant components observed in the XPS spectra of Fig. 1(a). The effect of each dopant $\mathrm{N}$ on the local electronic structure near the Fermi level of the surrounding carbon atoms are discussed later. In Figs. 1(c) and 1(d), some of the bright regions consist of the well-known superstructure of graphite formed by the standing wave of $\pi$ electrons around the graphite surface defects. ${ }^{39,40}$ In particular, this region does not spread isotropically around the defect but seems to propagate in three directions, as observed in the case of a single-atom vacancy defect on a graphite surface. ${ }^{30}$

\section{B. Pyridinic-N}

The experimentally observed STM image and STS spectrum of a type-A defect are shown in Fig. 2. The STS spectrum shown in Fig. 2(b) was measured at the position indicated by the arrow in Fig. 2(a), and it consists of a large peak around $-370 \mathrm{mV}$ and smaller peaks within a parabolic background. The spectrum is different from that of a single-atom vacancy with a single and large STS peak at ${ }^{41}$ or just above ${ }^{30,42}$ the Fermi level. The STS spectrum of the type-A defect is again different from that of the single-atom vacancy in graphite in terms of a propagation feature. ${ }^{30}$ That is, the modified electronic states of the single vacancy propagate anisotropically to three directions, while such an anisotropic propagation was not observed for the type-A defect, as shown in Figs. S6-S8 in the Supplemental Material. ${ }^{47}$ To identify the defect species in Fig. 2(a), STM images and STS spectra were simulated for several types of structures with pyridinic-N or graphitic- $\mathrm{N}$ species because these are the dominant species (a)

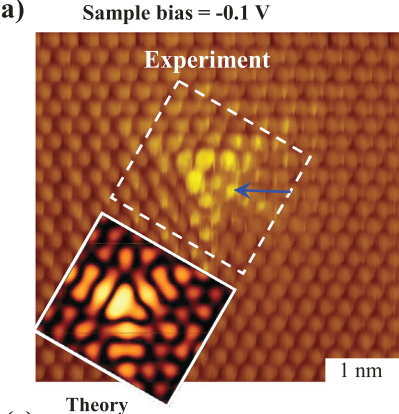

(c)

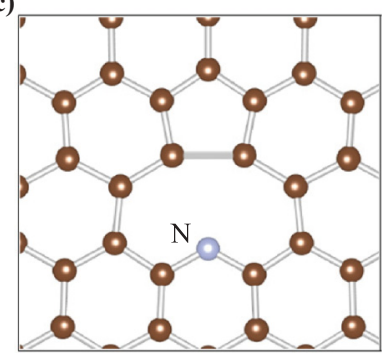

(b)

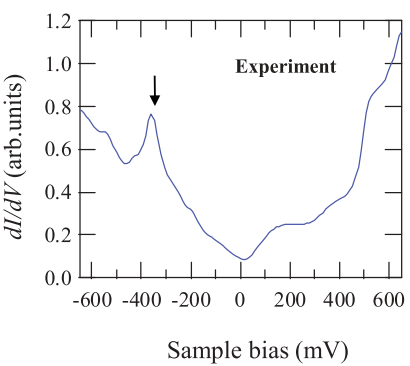

(d)

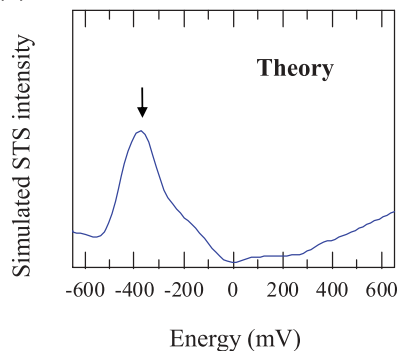

FIG. 2. (Color online) (a) STM topographic image of region A in Fig. 1(b) (scan size: $4.81 \times 4.61 \mathrm{~nm}^{2}, I_{t}=96.9 \mathrm{pA}, V_{s}=$ $-108 \mathrm{mV})$. The simulated STM image $(V=-0.1 \mathrm{~V})$ is also shown for comparison. (b) STS spectrum measured at the position indicated by the arrow in (a). (c) Equilibrium geometry of the pyridinic-N defect calculated by DFT. (d) Simulated STS spectrum of pyridinic-N.

in our sample, as shown by the XPS peak components in Fig. 1(a). After comparison with the results of different defect models, we found that the simulated STM and STS features of pyridinic-N well reproduced those measured in experiments, as shown in Fig. 2; i.e., the propagation of the bright region in STM and the appearance of the STS peak around $-370 \mathrm{mV}$ [Figs. 2(a) and 2(c)] are common between experiment and theory. The defect was thus assigned as pyridinic-N.

From the DFT calculation, the large STS peak at $-370 \mathrm{mV}$ in Figs. 2(b) and 2(d) can be assigned to localized $\pi$ states ( $p_{z}$ orbitals) because of the localized DOS character (see Fig. S2 in the Supplemental Material ${ }^{47}$ ). As shown in Fig. 3(a), the spatial propagation of the localized $\pi$ states can be seen in the STM simulations at a negative bias of $-1.0 \mathrm{~V}$. Most of the bright spots in Fig. 3(a) correspond to the LDOS for the STS peak at $-370 \mathrm{mV}$ (see Fig. S2 in the Supplemental Material ${ }^{47}$ ) and are located on few $\mathrm{C}$ atoms, part of the same sublattice hosting the $\mathrm{N}$ atom.

To further study the electronic structure near the nitrogen atom, the DFT equilibrium geometry and charge density isosurfaces are examined, as shown in Figs. 3(b) and 3(e). The isosurface stands for the spatial distribution of orbitals with certain electron densities, as described in Sec. III. First, the pentagonal ring is found to be formed by the rearrangement of the two "edge" carbon atoms facing the nitrogen atom, which is similar to the case of the $\mathrm{C}$ vacancy in graphene. ${ }^{43}$ Second, the $\mathrm{N}$ atom site is imaged as dark in the simulated STM image at $-1.0 \mathrm{~V}$ [Fig. 3(a)], even though the nitrogen atom should bear one more electron compared to carbon. This is explained by the occupation of four electrons in the $s p^{2}$ coplanar orbitals of $\mathrm{N}$ with a lone pair, as can be seen in Figs. 3(c) and 3(f), where 
(a) Sample bias $=-1.0 \mathrm{~V}$

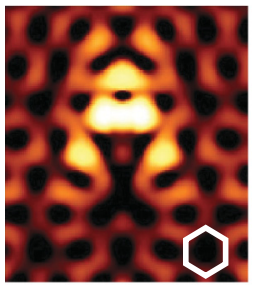

(b)

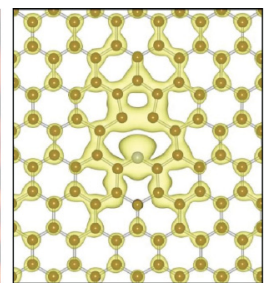

(e)

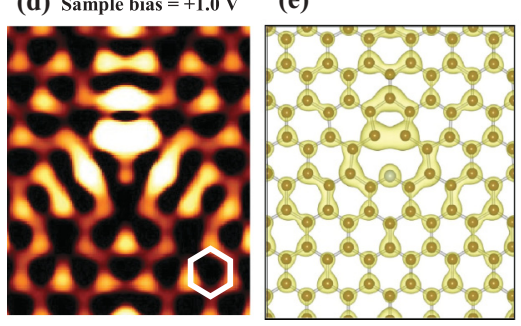

(c)

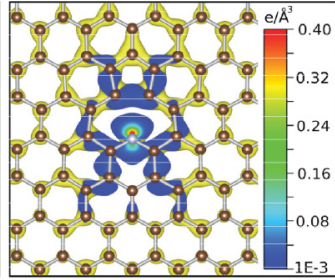

(f)

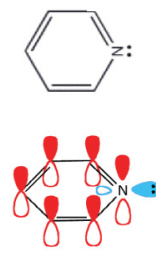

FIG. 3. (Color online) Simulated STM images (constant-current mode) for the pyridinic-N graphene defect: (a) $V=-1.0 \mathrm{~V}$ and (d) $V=+1.0 \mathrm{~V}$. The $\mathrm{N}$ atom is placed at the center of the image. (b) DFT equilibrium geometry and isosurface plot of electron density at $1.5 \times 10^{-3}$ electrons $/ \AA^{3}$ integrated from $-0.7 \mathrm{eV}$ to the Fermi level (square modulus of the wave function up to the Fermi level from -0.7 $\mathrm{eV}$ ). The $\mathrm{N}$ atom is shown in light gray. (c) Same image as Fig. 3(b) except for color near the nitrogen atom, where only the $x y$-plane DOS contribution near $\mathrm{N}$ is represented by blue-red gradients. (e) DFT equilibrium geometry and isosurface plot of electron density at $1.5 \times 10^{-3}$ electrons $/ \AA^{3}$ integrated from the Fermi level to +0.7 eV. (f) Schematic representation of $p_{z}$ orbitals in pyridinic-N.

the in-plane contribution to the electron density is represented by blue-red gradients.

Here, we discuss the appearance of the localized states in the occupied region. The formation of the lone pair suggests that the $\mathrm{N}$ atom of the pyridinic defect is negatively charged. The negatively charged $\mathrm{N}$ is consistent with the chemical shift in $\mathrm{N}$ $1 \mathrm{~s}$ binding energy to lower energy for pyridinic-N $(398.5 \mathrm{eV})$ in the XPS spectrum compared to that for graphitic-N $(401.1 \mathrm{eV})$. If $\mathrm{N}$ is negatively charged, the surrounding carbon atoms would be charged positively because of the screening effect. The positive charge of carbon can explain a shift of the localized $\pi$ state of carbon from the Fermi level to the lower (deeper) energy level. Following this picture, we suggest that the states corresponding to the STS peak in the occupied region near the Fermi level may act as a Lewis base toward molecular species.

At positive bias, i.e., for the unoccupied region, the isosurface plot in Fig. 3(e) shows a nodal plane across the pentagon, suggesting the antibonding character of the states lying above the pentagon ring. A slightly long-range propagating feature can be observed in the simulated STM image of Fig. 3(d), suggesting the appearance of localized states. There is, however, no distinct sharp peak in the simulated STS spectrum in the energy range from 0 to $+1.0 \mathrm{eV}$. The propagation can thus be explained by the presence of weakly localized $\pi$ states, where the localization is much weaker compared to the occupied region, as shown by the isosurface plot in Fig. 3(e). The feature of threefold propagation in Fig. 3(e) is similar to the case of a single-atom vacancy in
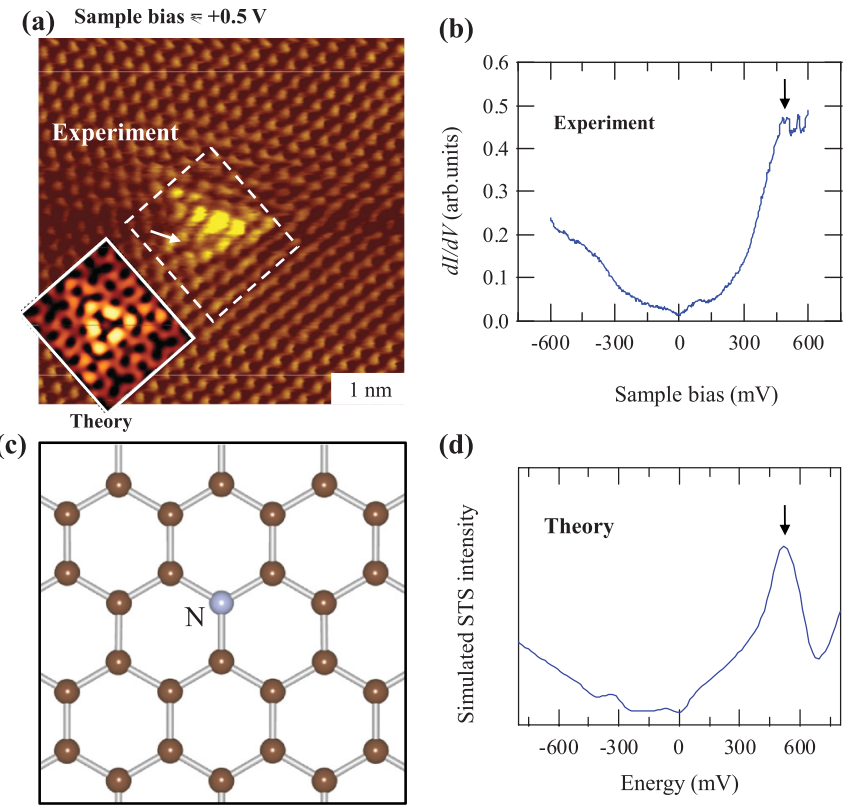

(d)

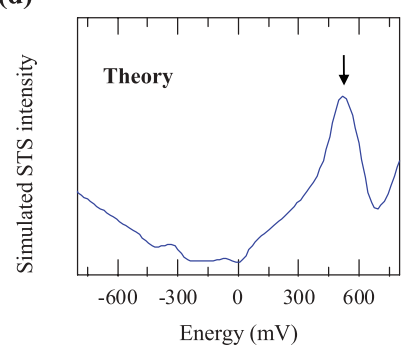

FIG. 4. (Color online) (a) STM topographic image corresponding to the defect shown in region $B$ in Fig. 1(b) (image taken from a different sample, scan size: $5.09 \times 5.08 \mathrm{~nm}^{2}, I_{t}=39.0 \mathrm{pA}, V_{s}=$ $500 \mathrm{mV})$. The simulated STM image $(\mathrm{V}=+0.5 \mathrm{~V})$ is also shown for comparison. (b) STS spectrum measured at the position indicated by the arrow in (a). (c) Equilibrium geometry of the graphitic- $\mathrm{N}$ defect calculated by DFT. (d) Simulated STS spectrum of graphitic-N.

graphite, ${ }^{30,41-44}$ though the localization is much weaker for pyridinic-N.

\section{Graphitic-N}

The experimentally observed STM image at $+500 \mathrm{mV}$ and STS spectrum of a type-B defect are shown in Fig. 4. The STS is different from that for the pristine graphite in terms of the asymmetric LDOS with respect to $0 \mathrm{~V}$ (the Fermi level), where larger intensity can be recognized in the positive bias region (unoccupied region). The small shoulder peak can be seen at the positive bias voltage, which is in contrast to that at the negative voltage observed for pyridinic-N. The STS spectra with the same shape have been measured in the vicinity of the defect independent of the lateral position, as measured in pyridinic-N (see Figs. S9-S14 ${ }^{47}$ ). To identify the defect species in Fig. 4(a), DFT simulations of STM images and STS spectra were carried out assuming several types of defect structures. After comparison with the results of different defect models, we found that the simulated STM and STS features of graphitic-N well reproduced those measured in experiments, as shown in Figs. 4 and S3. That is, triangular bright spots in the STM image and the STS peak (enhanced intensity) around $+500 \mathrm{mV}$ are common between experiment and theory. The defect was thus assigned as graphitic-N. In the DFT calculation, the position of the $\mathrm{N}$ atom in graphitic- $\mathrm{N}$ species is almost the same as that of the $\mathrm{C}$ atom in graphite (shorter by $0.002 \mathrm{~nm}$ for the $\mathrm{CN}$ bond length than for the $\mathrm{CC}$ bond length). That is, the nitrogen species takes the $s p^{2}$ planar structure of graphite. Concerning the origin of the STS peak at $500 \mathrm{mV}$, the state is ascribed to localized $\pi$ states ( $p_{z}$ orbitals), which 
(a) Sample bias $=-\mathbf{- 0 . 5} \mathrm{V}$

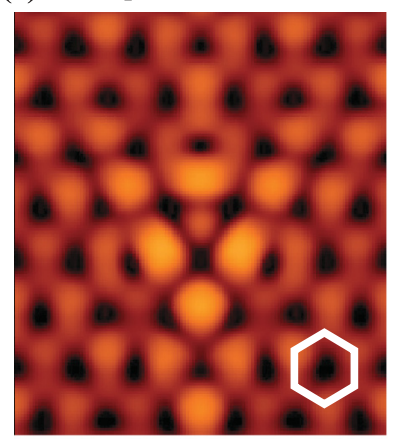

(c) Sample bias $=+0.5 \mathrm{~V}$

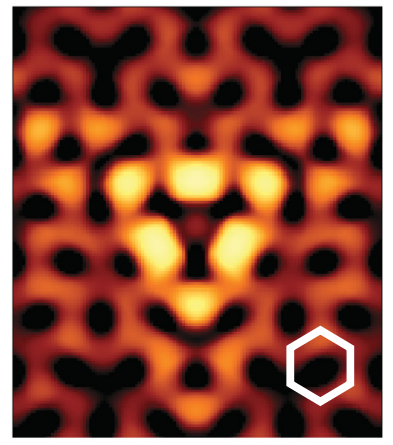

(b)

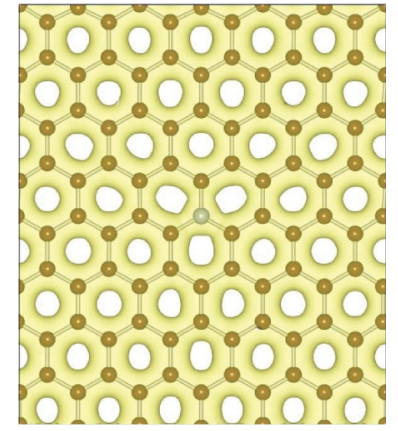

(d)

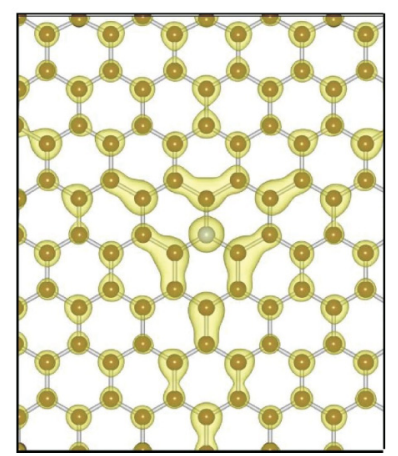

FIG. 5. (Color online) Simulated STM images of the graphitic-N defect: (a) $V=-0.5 \mathrm{~V}$, and (c) $V=+0.5 \mathrm{~V}$. The $\mathrm{N}$ atom is placed at the center of the image. DFT equilibrium geometry and isosurface plot of electron (b) electron density at $2 \times 10^{-4}$ electrons $/ \AA^{3}$ integrated from $-0.7 \mathrm{eV}$ to the Fermi level (square modulus of wave function up to the Fermi level from $-0.7 \mathrm{eV}$ ) and (d) electron density at $1.5 \times$ $10^{-3}$ electrons $/ \AA^{3}$ integrated from the Fermi level to $+0.7 \mathrm{eV}$.

is similar to the edge state because of the localized character (see Fig. S3 in the Supplemental Material ${ }^{47}$ ). In the simulated STM image (Fig. 5), the bright triangular spots are due to the enhanced LDOS component around $+500 \mathrm{mV}$. In addition, 15-20 carbon atoms are imaged bright, indicating that the localized $\pi$ states propagate near the defect. As shown in Figs. S4 and $\mathrm{S} 5,{ }^{47}$ the $\mathrm{N}$ atom position in Fig. 4(a) is found as the substituted position of a $\beta$-carbon atom $(\mathrm{C}$ atoms located above the center of a hexagonal ring; the remaining $\mathrm{C}$ atoms are called $\alpha$-carbon atoms ${ }^{45}$ ).

Here, we discuss the appearance of the localized states in the unoccupied region. The positively charged $\mathrm{N}$ is expected by the chemical shift in $\mathrm{N}$ 1s binding energy to higher energy for graphitic-N (401.1 eV) in the XPS spectrum compared to that for pyridinic-N (398.5 eV). Indeed, the difference of $2.6 \mathrm{eV}$ is so large that the binding energy of $401.1 \mathrm{eV}$ is comparable with that for the pyridinium ion, $\mathrm{C}_{5} \mathrm{H}_{5} \mathrm{NH}^{+}(401.2 \mathrm{eV})$, and the ammonium ion, $\mathrm{NH}_{4}^{+}(401.5 \mathrm{eV})$, where $\mathrm{N}$ is positively charged in both cases, ${ }^{27}$ suggesting a positive charge for $\mathrm{N}$ of graphitic-N. The positively charged $\mathrm{N}$ of graphitic-N has also been reported recently based on theoretical results by Yu et al. ${ }^{8}$ and Meyer et al. ${ }^{46}$ The positive charge can be explained by electron transfer from the $\mathrm{N}$ atom to the $\pi$ conjugated state. If $\mathrm{N}$ is positively charged, the surrounding carbon atoms should be charged negatively because of the screening effect. The negative charge of carbon can explain a shift of the localized $\pi$ state of carbon from the Fermi level to the upper energy level.

In addition, the screening of the positive $\mathrm{N}$ charge may be the source of the dark halo around the graphitic-N defect seen in Figs. 1(b) and 1(d); i.e., the charge density rearrangement may be induced by the positive charge of $\mathrm{N}$, and it may cause the modulated contrast in the STM image around the $\mathrm{N}$ atom. Here, the states with the STS peak in the unoccupied region near the Fermi level may act as a Lewis acid. The STS peak position of graphitic-N should depend on the amount of doped nitrogen. The amount of electron doping $(n)$ due to $\mathrm{N}$ impurities per unit area in a single graphene layer can be approximately quantified by the integral of the new (shifted) Fermi surface $(F S)$ at a given energy $(E)$ away from the pristine Fermi level. This, for independent electrons, is represented as

$$
n=\frac{4}{4 \pi^{2}} \oint_{F S} d \mathbf{k}=\frac{|k|^{2}}{\pi}=\frac{E^{2}}{\hbar^{2} \pi v_{F}^{2}}
$$

in which we included the factor 4 to account for spin and valley degeneracy and where the linear band dispersion of graphene $E=\hbar v_{F}|k|$ was used. Then, the shift in the Fermi energy should scale as $E=\hbar v_{F}(\pi n)^{1 / 2}$, where $v_{F}$ is the Fermi velocity $\left(\sim 10^{6} \mathrm{~m} \mathrm{~s}^{-1}\right)$. Following these arguments, it may be possible to control the population of the defect states by controlling the $\mathrm{N}$ concentration. For low $\mathrm{N}$ doses (very small $n$ ), the extra electron introduced by the impurity into the graphene layer would cause infinitely small doping, thus inducing a positive charge on the $\mathrm{N}$ atom. Otherwise, for high doses, the doping would be sufficient to doubly occupy the $p_{z}$ orbital (i.e., the states responsible for the STS peak at +500 $\mathrm{mV}$ ), giving rise to a neutral defect. From Eq. (1), we estimated that a concentration of more than $1.8 \times 10^{13}$ defects $/ \mathrm{cm}^{2}$ $(\sim 1$ graphitic-N for every 210 carbon atoms in the single graphene layer) should be sufficient to raise the Fermi energy by $\sim 0.5 \mathrm{eV}$ where these states occur.

\section{CONCLUSIONS}

We have examined a N-doped graphite surface by STM, STS, XPS, and first-principles calculations. Two types of nitrogen species have been identified on the $\mathrm{N}$-doped graphite surface: pyridinic- $\mathrm{N}$ with single-atom vacancy and graphitic$\mathrm{N}$. While pyridinic-N defects induce an atomic rearrangement to form a pentagon, graphitic-N affects the structure only slightly. In each case, the electronic structure of graphite close to the Fermi energy is found to be modified by the defects. The distinct localized $\pi$ states appear in occupied and unoccupied regions near the Fermi level around pyridinic-N and graphitic-N species, respectively.

\section{ACKNOWLEDGMENTS}

This work was financially supported by the New Energy and Industrial Technology Development Organization of Japan. We acknowledge CINECA consortium for computational resources and Rocco Martinazzo for fruitful discussions. 
*Present address: Toyota Motor. Corporation, 1200, Mishuku, Susono, Shizuoka, 410-11 Japan.

${ }^{\dagger}$ Corresponding author: nakamura@ims.tsukuba.ac.jp

${ }^{1}$ M. Deifallah, P. F. McMillan, and F. Cora, J. Phys. Chem. C 112, 5447 (2008).

${ }^{2}$ N. M. R. Peres, F. Guinea, and A. H. CastroNeto, Phys. Rev. B 72, 174406 (2005).

${ }^{3}$ S. Stafström, Appl. Phys. Lett. 77, 3941 (2000).

${ }^{4}$ A. A. Thomas, A. Fischer, F. Goettmann, M. Antonietti, J. O. Müller, R. Schlögl, and J. M. Carlsson, J. Mater. Chem. 18, 4893 (2008).

${ }^{5}$ S. S. Yu, W. T. Zheng, Q. B. Wen, and Q. Jiang, Carbon 46, 537 (2008).

${ }^{6}$ S. Maldonado, S. Morin, and K. J. Stevenson, Carbon 44, 1429 (2006).

${ }^{7}$ T. Kondo, T. Suzuki, and J. Nakamura, J. Phys. Chem. Lett. 2, 577 (2011).

${ }^{8}$ S.-S. Yu and W.-T. Zheng, Nanoscale 2, 1069 (2010).

${ }^{9}$ Y. Wang, Y. Shao, D. W. Matson, J. Li, and Y. Lin, ACS Nano 4, 1790 (2010).

${ }^{10}$ L. Qu, Y. Liu, J.-B. Baek, and L. Dai, ACS Nano 4, 1321 (2010).

${ }^{11}$ S. F. Huang, K. Terakura, T. Ozaki, T. Ikeda, M. Boero, M. Oshima, J. I. Ozaki, and S. Miyata, Phys. Rev. B 80, 235410 (2009).

${ }^{12}$ C. V. Rao, C. R. Cabrera, and Y. Ishikawa, J. Phys. Chem. Lett. 1, 2622 (2010).

${ }^{13}$ Y. Zhou, J. Mater. Chem. 19, 7830 (2009).

${ }^{14}$ R. A. Sidik, A. B. Anderson, N. P. Subramanian, S. P. Kumaraguru, and B. N. Popov, J. Phys. Chem. B 110, 1787 (2006).

${ }^{15}$ D. H. Lee, W. J. Lee, W. J. Lee, S. O. Kim, and Y.-H. Kim, Phys. Rev. Lett. 106, 175502 (2011).

${ }^{16}$ H. M. Jeong, J. W. Lee, W. H. Shin, Y. J. Choi, H. J. Shin, J. K. Kang, and J. W. Choi, Nano Lett. 11, 2472 (2011).

${ }^{17}$ X. Wang, X. Li, L. Zhang, Y. Yoon, P. K. Weber, H. Wang, J. Guo, and H. Dai, Science 324, 768 (2009).

${ }^{18}$ R. A. Nistor, D. M. Newns, and G. J. Martyna, ACS Nano 5, 3096 (2011).

${ }^{19}$ D. Wei, Y. Liu, Y. Wang, H. Zhang, L. Haung, and G. Yu, Nano Lett. 9, 1752 (2009).

${ }^{20}$ Y. Li, Z. Zhou, P. Shen, and Z. Chen, ACS Nano 3, 1952 (2009).

${ }^{21}$ B. Biel, X. Blase, F. Triozon, and S. Roche, Phys. Rev. Lett. 102, 096803 (2009).

${ }^{22}$ G. Imamura and K. Saiki, J. Phys. Chem. C 115, 10000 (2011).

${ }^{23}$ K.-J. Kim, H. Lee, J. Choi, H. Lee, M. C. Jung, H. J. Shin, T. H. Kang, B. Kim, and S. Kim, J. Phys. Condens. Matter 22, 045005 (2010)

${ }^{24}$ D. Deng, X. Pan, L. Yu, Y. Cui, Y. Jiang, J. Qi, W. Li, Q. Fu, X. Ma, Q. Xue, G. Sun, and X. Bao, Chem. Mater. 23, 1188 (2011).
${ }^{25}$ D.-Q. Yang and E. A. Sacher, Surf. Sci. 531, 185 (2003).

${ }^{26}$ I. Kusunoki, M. Sakai, Y. Igari, S. Ishidzuka, T. Takami, T. Takaoka, M. N. Gamo, and T. Ando, Surf. Sci. 492, 315 (2001).

${ }^{27}$ J. R. Pels, F. Kapteijn, J. A. Moulijn, Q. Zhu, and K. M. Thomas, Carbon 33, 1641 (1995).

${ }^{28}$ E. Raymundo-Piñero, D. Cazorla-Amorós, A. Linares-Solano, J. Find, U. Wild, and R. Schlögl, Carbon 40, 597 (2002).

${ }^{29}$ R. Arrigo, M. Hävecker, S. Wrabetz, R. Blume, M. Lerch, J. McGregor, E. Parrott, J. Zeitler, L. Gladden, A. Knop-Gericke, R. Schlögl, and D. S. Su, J. Am. Chem. Soc. 132, 9616 (2010).

${ }^{30}$ T. Kondo, Y. Honma, J. Oh, T. Machida, and J. Nakamura, Phys. Rev. B 82, 153414 (2010).

${ }^{31}$ J. Tersoff and D. R. Hamann, Phys. Rev. B 31, 805 (1985).

${ }^{32}$ S. Casolo, R. Martinazzo, and G. F. Tantardini, J. Phys. Chem. C 115, 3250 (2011).

${ }^{33}$ G. Kresse and J. Furthmüller, Comput. Mat. Sci. 6, 15 (1996).

${ }^{34}$ P. E. Blöchl, Phys. Rev. B 50, 17953 (1994).

${ }^{35}$ G. Kresse and D. Joubert, Phys. Rev. B 59, 1758 (1999).

${ }^{36}$ J. P. Perdew, K. Burke, and M. Ernzerhof, Phys. Rev. Lett. 77, 3865 (1996).

${ }^{37}$ J. M. Soler, E. Artacho, J. D. Gale, A. García, J. Junquera, P. Ordejón, and D. Sánchez-Portal, J. Phys. Condens. Matter 14, 2745 (2002).

${ }^{38}$ D.-Q. Yang and E. Sacher, App. Surf. Sci. 195, 202 (2002).

${ }^{39} \mathrm{P}$. Ruffieux, M. Melle-Franco, O. Groning, M. Bielmann, F. Zerbetto, and P. Groning, Phys. Rev. B 71, 153403 (2005).

${ }^{40}$ H. A. Mizes and J. S. Foster, Science 244, 559 (1989).

${ }^{41}$ M. M. Ugeda, I. Brihuega, F. Guinea, and J. M. Gomez-Rodriguez, Phys. Rev. Lett. 104, 096804 (2010).

${ }^{42}$ J. Oh, T. Kondo, D. Hatake, Y. Honma, K. Arakawa, T. Machida, and J. Nakamura, J. Phys. Condens. Matter 22, 304008 (2010).

${ }^{43}$ Y. Ma, P. O. Lehtinen, A. S. Foster, and R. M. Nieminen, New J. Phys. 6, 68 (2004)

${ }^{44}$ R. Martinazzo, S. Casolo, and G. F. Tantardini, in Physics and Applications of Graphene-Theory, edited by S. Mikhailov (Intech, Rijeka, 2011), Chap. 3, pp. 45.

${ }^{45}$ D. Tomanek, S. G. Louie, H. J. Mamin, D. W. Abraham, R. E. Thomson, E. Ganz, and J. Clarke, Phys. Rev. B 35, 7790 (1987).

${ }^{46}$ J. C. Meyer, S. Kurasch, H. J. Park, V. Skakalova, D. Kuenzel, A. Gross, A. Chuvilin, G. Algara-Siller, S. Roth, T. Iwasaki, U. Starke, J. H. Smet, and U. Kaiser, Nat. Mater. 10, 209 (2011).

${ }^{47}$ See Supplemental Material at http://link.aps.org/supplemental/ 10.1103/PhysRevB.86.035436 for efficiency of N-doping, effect of the sample bias on the STM images, analysis and effect of $\mathrm{N}$ atom position, STS around defect of nitrogen doped graphite and example of the characterization of $\mathrm{N}$ species on $\mathrm{N}$-doped graphite based on the current work. 